Homology, Homotopy and Applications, vol.12(1), 2010, pp.357-366

\title{
ON TRIVIALITIES OF STIEFEL-WHITNEY CLASSES OF VECTOR BUNDLES OVER ITERATED SUSPENSION SPACES
}

\author{
RYUICHI TANAKA
}

\author{
(communicated by Brooke Shipley)
}

\begin{abstract}
A space $B$ is described as $\mathrm{W}$-trivial if for every vector bundle over $B$, all the Stiefel-Whitney classes vanish. We prove that if $B$ is a 9 -fold suspension, then $B$ is $\mathrm{W}$-trivial. We also determine all pairs $(k, n)$ of positive integers for which $\Sigma^{k} F P^{n}$ is W-trivial, where $F=\mathbb{R}, \mathbb{C}$ or $\mathbb{H}$.
\end{abstract}

\section{Introduction and results}

A space $B$ is called $\mathrm{W}$-trivial if $W(\alpha)=1$ holds for every vector bundle $\alpha$ over $B$. Here $W(\alpha)$ denotes the total Stiefel-Whitney class of $\alpha$. If $B$ is $\mathrm{W}$-trivial, then a kind of Borsuk-Ulam type theorem holds for every vector bundle $\alpha$ over $B$; precisely, for any integer $i$ with $i>\operatorname{dim} \alpha$, there does not exist a $\mathbb{Z}_{2}$-map from $S^{i-1}$ to $S(\alpha)$, the sphere bundle of $\alpha$ [6, Proposition 2.2]. Thus it would be interesting to ask whether a space is $\mathrm{W}$-trivial or not. As is well-known, the sphere $S^{n}$ is $\mathrm{W}$-trivial if and only if $n \neq 1,2,4,8$ (see [5]). Obviously, the projective space $F P^{n}$, where $F=\mathbb{R}, \mathbb{C}$ or $\mathbb{H}$, is not W-trivial for any $n>0$. For the stunted projective space $F P_{m}^{n}$, all $(m, n)$ for which $F P_{m}^{n}$ is W-trivial were determined in [9]; roughly speaking, $F P_{m}^{n}$ is not W-trivial if and only if $m$ is very small compared with $n$.

As is seen in the case $B=S^{n}$, it is not true that if $B$ is W-trivial, then its suspension $\Sigma B$ is also $\mathrm{W}$-trivial. In this paper, we first prove the following theorem.

Theorem 1.1. For a space B, its 8-fold suspension $\Sigma^{8} B$ is $W$-trivial if either of the following conditions is satisfied:

(1) $B$ is $W$-trivial.

(2) The cup product in $\widetilde{H}^{*}\left(B ; \mathbb{Z}_{2}\right)$ is trivial.

In general, the cup product in $\widetilde{H}^{*}\left(\Sigma B ; \mathbb{Z}_{2}\right)$ is trivial, so that from the above theorem, we immediately obtain the following result.

Corollary 1.2. For any space $B$, its 9 -fold suspension $\Sigma^{9} B$ is $W$-trivial.

As is easily seen by using the suspension theorem, a $k$-connected complex $B$ with $\operatorname{dim} B \leqslant 2 k+1$ is homotopy equivalent to the suspension of a $(k-1)$-connected complex of dimension $\operatorname{dim} B-1$. By iterating this, we see that a $k$-connected complex

Received March 15, 2010, revised March 28, 2010; published on May 3, 2010.

2000 Mathematics Subject Classification: 55R50, 55S05.

Key words and phrases: Stiefel-Whitney class, vector bundle, squaring operation.

This article is available at http://intlpress.com/HHA/v12/n1/a18

Copyright (c) 2010, International Press. Permission to copy for private use granted. 
$B$ is homotopy equivalent to the 9 -fold suspension of a $(k-9)$-connected complex $(k>9)$ if $\operatorname{dim} B \leqslant 2 k-7$. Therefore, from Corollary 1.2 , we obtain the following result.

Corollary 1.3. Let $B$ be a $k$-connected complex with $k>9$. If $\operatorname{dim} B \leqslant 2 k-7$, then $B$ is $W$-trivial.

This corollary greatly improves Theorem 1.3 in [8]. Since the smallest integer $i$ such that $w_{i}(\alpha) \neq 0$ is a power of 2 (see [8, Lemma 2.1]), the above corollary is actually useful only when $k \geqslant 12$. For example, we see that a 16 -dimensional complex is $\mathrm{W}$-trivial if it is 12-connected. It should be also noted that the 16-dimensional stunted projective space $\mathbb{R} P_{k}^{16}$ is $\mathrm{W}$-trivial for $9<k<16$ while $\mathbb{R} P_{9}^{16}$ is not W-trivial (see [8, Theorem 4.1]).

Next, in this paper, we investigate whether $\Sigma^{k} F P^{n}$ is $\mathrm{W}$-trivial or not, where $F=\mathbb{R}, \mathbb{C}$ or $\mathbb{H}$. Because of Corollary 1.2 , our interests are only in the case when $0<k \leqslant 8$. For $F=\mathbb{R}$, we have the following result.

Theorem 1.4. For positive integers $k$ and $n$, the $k$-fold suspension $\Sigma^{k} \mathbb{R} P^{n}$ of $\mathbb{R} P^{n}$ is not $W$-trivial if and only if $k$ and $n$ satisfy one of the following conditions:

(1) $k=1,2,4$ or 8 and $n \geqslant k$.

(2) $k=3,5$ or 7 and $n+k=4$ or 8 .

(3) $k=6$ and $n=2$ or 3 .

This result shows that the condition $k \geqslant 9$ is best possible for $\Sigma^{k} B$ to be W-trivial in general.

For $F=\mathbb{C}$ and $F=\mathbb{H}$, we have the following results.

Theorem 1.5. For positive integers $k, n$ with $n>1$, the $k$-fold suspension $\Sigma^{k} \mathbb{C} P^{n}$ of $\mathbb{C} P^{n}$ is not $W$-trivial if and only if $k=2$ or 4 .

Theorem 1.6. For positive integers $k, n$ with $n>1$, the $k$-fold suspension $\Sigma^{k} \mathbb{H} P^{n}$ of $\mathbb{H} P^{n}$ is not $W$-trivial if and only if $k=4$.

It is worth noting that the W-triviality of $\Sigma^{k} F P^{n}$ does not depend on $n$ for $F=\mathbb{C}$ or $\mathbb{H}$.

Throughout this paper, all cohomology is assumed to have coefficients $\mathbb{Z}_{2}$ unless otherwise stated. The total Stiefel-Whitney class of $\alpha$ is denoted by $W(\alpha)$, and the total Chern class by $C(\alpha)$.

The following two lemmas are straightforward to show but they are of fundamental importance for our proofs of theorems.

\section{Lemma 1.7.}

(1) If $\widetilde{K O}(B)=0$, then $B$ is W-trivial.

(2) Let $f: B \rightarrow X$ be a map and suppose that $X$ is W-trivial. If $f^{*}: \widetilde{K O}(X)$ $\rightarrow \widetilde{K O}(B)$ is epimorphic, then $B$ is W-trivial.

\section{Lemma 1.8.}

(1) If $H^{2^{r}}(B)=0$ for all $r \geqslant 0$, then $B$ is $W$-trivial.

(2) Let $f: X \rightarrow B$ be a map and suppose that $X$ is $W$-trivial. If $f^{*}: H^{2^{r}}(B)$ $\rightarrow H^{2^{r}}(X)$ is monomorphic for all $r \geqslant 0$, then $B$ is $W$-trivial. 


\section{Proof of Theorem 1.1}

In this section, we prove Theorem 1.1. We use the Bott periodicity theorem for $K O$-theory. Let $j: S^{8} \times B \rightarrow \Sigma^{8} B$ denote the quotient map and let $p_{1}: S^{8} \times B \rightarrow S^{8}$ and $p_{2}: S^{8} \times B \rightarrow B$ denote the projections. Let $\alpha$ be an arbitrary vector bundle over $\Sigma^{8} B$. By the Bott periodicity theorem, we see that $j^{*} \alpha$ is stably equivalent to $p_{1}^{*}(\nu-8) \otimes p_{2}^{*}(\beta-m)$ for some vector bundle $\beta$ over $B$. Here $\nu$ denotes the Hopf vector bundle over $S^{8}$ and $m=\operatorname{dim} \beta$. Then, we have

$$
j^{*} W(\alpha)=W\left(p_{1}^{*} \nu \otimes p_{2}^{*} \beta\right) \cdot W\left(p_{1}^{*} \nu\right)^{-m} \cdot W\left(p_{2}^{*} \beta\right)^{-8} .
$$

We compute this and show that $W(\alpha)=1$. Note that $W\left(p_{1}^{*} \nu\right)=p_{1}^{*} W(\nu)=1+s \times 1$, where $s$ denotes the generator of $H^{8}\left(S^{8}\right)$. Let

$$
W\left(p_{1}^{*} \nu\right)=\prod_{i=1}^{8}\left(1+s_{i}\right) \quad \text { and } \quad W\left(p_{2}^{*} \beta\right)=\prod_{j=1}^{m}\left(1+t_{j}\right)
$$

be formal factorizations of $W\left(p_{1}^{*} \nu\right)$ and $W\left(p_{2}^{*} \beta\right)$. Then, by an analogous formula to Formula III of Theorem 4.4.3 in [4], we have $W\left(p_{1}^{*} \nu \otimes p_{2}^{*} \beta\right)=\prod_{i, j}\left(1+s_{i}+t_{j}\right)$. We first calculate the product for $i$ 's by using $\prod_{i=1}^{8}\left(1+s_{i}\right)=1+s \times 1$ as follows:

$$
\begin{aligned}
\prod_{i=1}^{8}\left(\left(1+t_{j}\right)+s_{i}\right) & =\sum_{k=0}^{8}\left(1+t_{j}\right)^{8-k} \lambda_{k}\left(s_{1}, s_{2}, \ldots, s_{8}\right) \\
& =\left(1+t_{j}\right)^{8}+s_{1} s_{2} \cdots s_{8} \\
& =1+t_{j}^{8}+s \times 1,
\end{aligned}
$$

where $\lambda_{k}$ denotes the elementary symmetric polynomial of degree $k$ and we used the fact that $\lambda_{k}\left(s_{1}, s_{2}, \ldots, s_{8}\right)=0$ for $0<k<8$. Therefore we have

$$
\begin{aligned}
W\left(p_{1}^{*} \nu \otimes p_{2}^{*} \beta\right) & =\prod_{j=1}^{m}\left((1+s \times 1)+t_{j}^{8}\right) \\
& =\sum_{k=0}^{m}(1+s \times 1)^{m-k} \lambda_{k}\left(t_{1}^{8}, t_{2}^{8}, \ldots, t_{m}^{8}\right) .
\end{aligned}
$$

Now, we assume that the cup product in $\widetilde{H}^{*}(B)$ is trivial. Then, we clearly have $W(\beta)^{2}=1$, so that $W\left(p_{2}^{*} \beta\right)^{8}=p_{2}^{*} W(\beta)^{8}=1$. This implies that $\prod_{j=1}^{m}\left(1+t_{j}^{8}\right)=1$, so that $\lambda_{k}\left(t_{1}^{8}, t_{2}^{8}, \ldots, t_{m}^{8}\right)=0$ for every $k>0$. Therefore we have

$$
W\left(p_{1}^{*} \nu \otimes p_{2}^{*} \beta\right)=(1+s \times 1)^{m} .
$$

Substituting these results into $(*)$, we obtain

$$
j^{*} W(\alpha)=(1+s \times 1)^{m} \cdot(1+s \times 1)^{-m} \cdot 1^{-1}=1 .
$$

Since $j^{*}: H^{*}\left(\Sigma^{8} B\right) \rightarrow H^{*}\left(S^{8} \times B\right)$ is monomorphic, we conclude that $W(\alpha)=1$. Thus the proof of Theorem 1.1 under the assumption (2) is completed.

The proof under the assumption (1) is quite similar. Since $W\left(p_{2}^{*} \beta\right)=1$ from the assumption that $B$ is $\mathrm{W}$-trivial, we may regard all the $t_{j}$ 's as zeros in our previous calculations. Then we obtain $W\left(p_{1}^{*} \nu \otimes p_{2}^{*} \beta\right)=(1+s \times 1)^{m}$ and have the same result as $(* *)$. Thus the theorem under the assumption (1) follows. 
Here we prepare the following lemma, which will be used to prove Theorems 1.4 and 1.5 in later sections.

Lemma 2.1. Let $d$ and $m$ be positive integers with $d \leqslant m$.

(1) If $\gamma$ is a vector bundle over $S^{d}$ with $\operatorname{dim} \gamma=m$ and $\beta$ is a line bundle over $B$, then in $H^{*}\left(S^{d} \times B\right)$ we have

$$
W\left(\left(p_{1}^{*} \gamma-m\right) \otimes\left(p_{2}^{*} \beta-1\right)\right)=1+w_{d}(\gamma) \times\left(\left(1+w_{1}(\beta)\right)^{-d}-1\right),
$$

where $p_{1}: S^{d} \times B \rightarrow S^{d}$ and $p_{2}: S^{d} \times B \rightarrow B$ are the projections.

(2) If $\gamma$ is a complex vector bundle over $S^{2 d}$ with $\operatorname{dim}_{\mathbb{C}} \gamma=m$ and $\beta$ is a complex line bundle over $B$, then in $H^{*}\left(S^{2 d} \times B ; \mathbb{Z}\right)$ we have

$$
C\left(\left(p_{1}^{*} \gamma-m\right) \otimes_{\mathbb{C}}\left(p_{2}^{*} \beta-1\right)\right)=1+c_{d}(\gamma) \times\left(\left(1+c_{1}(\beta)\right)^{-d}-1\right),
$$

where $p_{1}: S^{2 d} \times B \rightarrow S^{2 d}$ and $p_{2}: S^{2 d} \times B \rightarrow B$ are the projections.

Proof. We prove only (1) since the proof of (2) is quite similar. Let us put $w_{d}(\gamma)$ $=s$ and $w_{1}(\beta)=t$. Let $W\left(p_{1}^{*} \gamma\right)=1+s \times 1=1^{m-d} \cdot \prod_{i=1}^{d}\left(1+s_{i}\right)$ and $W\left(p_{2}^{*} \beta\right)=$ $1+1 \times t=1+t_{1}$ be formal factorizations. Then, just like before, we can calculate as follows:

$$
\begin{aligned}
W\left(p_{1}^{*} \gamma \otimes p_{2}^{*} \beta\right) & =\left(1+t_{1}\right)^{m-d} \cdot \prod_{i=1}^{d}\left(1+s_{i}+t_{1}\right) \\
& =\left(1+t_{1}\right)^{m-d} \cdot\left(\left(1+t_{1}\right)^{d}+s \times 1\right) \\
& =(1+1 \times t)^{m} \cdot\left(1+s \times(1+t)^{-d}\right)
\end{aligned}
$$

Therefore, we have

$$
\begin{aligned}
W\left(\left(p_{1}^{*} \gamma-m\right) \otimes\left(p_{2}^{*} \beta-1\right)\right) & =W\left(p_{1}^{*} \gamma \otimes p_{2}^{*} \beta\right) \cdot W\left(p_{2}^{*} \beta\right)^{-m} \cdot W\left(p_{1}^{*} \gamma\right)^{-1} \\
& =\left(1+s \times(1+t)^{-d}\right) \cdot(1+s \times 1)^{-1} \\
& =\left(1+s \times(1+t)^{-d}\right) \cdot(1-s \times 1) \\
& =1+s \times\left((1+t)^{-d}-1\right) .
\end{aligned}
$$

Thus the lemma follows.

\section{Proof of Theorem 1.4}

In this section, we investigate whether $\Sigma^{k} \mathbb{R} P^{n}$ is W-trivial or not. Since $\Sigma^{k} \mathbb{R} P^{n}$ is W-trivial for $k>8$ by Corollary 1.2 , our interests are only in the case when $0<k \leqslant 8$. We divide into three cases: (1) $k=1,2,4$ or 8 , (2) $k=3,5$ or 7 and (3) $k=6$.

First we consider the case when $k=1,2,4$ or 8 . The result is as follows.

Proposition 3.1. Let $d=1,2,4$ or 8 . Then $\Sigma^{d} \mathbb{R} P^{n}$ is not $W$-trivial if and only if $n \geqslant d$.

Proof. Recall that for a vector bundle $\alpha$, the smallest integer $i$ such that $w_{i}(\alpha) \neq 0$ is a power of 2 (see [8, Lemma 2.1]). If $n<d$, then $\Sigma^{d} \mathbb{R} P^{n}$ has no cells of dimension a power of 2 , so that $\Sigma^{d} \mathbb{R} P^{n}$ is $\mathrm{W}$-trivial. Now, let us consider the exact sequence

$$
0 \longleftarrow \widetilde{K O}\left(S^{d} \vee \mathbb{R} P^{n}\right) \stackrel{i^{*}}{\longleftarrow} \widetilde{K O}\left(S^{d} \times \mathbb{R} P^{n}\right) \stackrel{j^{*}}{\longleftarrow} \widetilde{K O}\left(\Sigma^{d} \mathbb{R} P^{n}\right) \longleftarrow 0
$$


where $i$ and $j$ are obvious maps. Let $\nu$ denote the Hopf vector bundle over $S^{d}$ and let $\xi$ denote the canonical line bundle over $\mathbb{R} P^{n}$. Since $i^{*}\left(\left(p_{1}^{*} \nu-d\right) \otimes\left(p_{2}^{*} \xi-1\right)\right)$ $=0$, there is a vector bundle $\alpha$ over $\Sigma^{d} \mathbb{R} P^{n}$ such that $j^{*} \alpha$ is stably equivalent to $\left(p_{1}^{*} \nu-d\right) \otimes\left(p_{2}^{*} \xi-1\right)$. By Lemma 2.1, in $H^{*}\left(S^{d} \times \mathbb{R} P^{n}\right)$ we have

$$
\begin{aligned}
W\left(j^{*} \alpha\right) & =1+s \times\left((1+t)^{-d}-1\right) \\
& =1+s \times\left(t^{d}+t^{2 d}+t^{3 d}+\cdots\right),
\end{aligned}
$$

where $s$ and $t$ denote the generator of $H^{d}\left(S^{d}\right)$ and $H^{1}\left(\mathbb{R} P^{n}\right)$ respectively. Hence, we see that $j^{*} W(\alpha) \neq 1$ if $n \geqslant d$. We thus conclude that $W(\alpha) \neq 1$, so that $\Sigma^{d} \mathbb{R} P^{n}$ is not W-trivial if $n \geqslant d$.

Before we consider the second case, we prepare a few lemmas.

Lemma 3.2. If $\Sigma^{k} \mathbb{R} P^{2^{m}-k}$ is $W$-trivial, then $\Sigma^{k} \mathbb{R} P^{n}$ is $W$-trivial for any integer $n$ with $2^{m}-k<n<2^{m+1}-k$.

Proof. Let $i: \Sigma^{k} \mathbb{R} P^{2^{m}-k} \rightarrow \Sigma^{k} \mathbb{R} P^{n}$ be the inclusion map. If $2^{m}<n+k<2^{m+1}$, then $i^{*}: H^{2^{r}}\left(\Sigma^{k} \mathbb{R} P^{n}\right) \rightarrow H^{2^{r}}\left(\Sigma^{k} \mathbb{R} P^{2^{m}-k}\right)$ is monomorphic for all $r \geqslant 0$ for dimensional reasons. Therefore, the lemma follows from Lemma 1.8.

Lemma 3.3. Let $\alpha$ be a vector bundle over a complex B. Let $r$ be an integer with $r \geqslant 2$ and suppose that $w_{i}(\alpha)=0$ for $0<i<2^{r}$. Then we have $\operatorname{Sq}^{j} w_{2^{r}}(\alpha)=0$ for $0<j<2^{r-1}$.

Proof. We put $2^{r-1}=m$ and consider the inclusion $i: B^{(3 m)} \hookrightarrow B$, where $B^{(3 m)}$ is the $3 m$-skeleton of $B$. For dimensional reasons, the induced bundle $i^{*} \alpha$ is stably equivalent to some $3 m$-dimensional vector bundle $\beta$. Then we clearly have $W\left(i^{*} \alpha\right)$ $=W(\beta)$. We denote by $P(\beta)$ the associated projective bundle of $\beta$, and by $e$ the $\mathbb{Z}_{2}$-Euler class of the line bundle $\beta \rightarrow P(\beta)$. The cohomology $H^{*}(P(\beta))$ is a free $H^{*}\left(B^{(3 m)}\right)$-module generated by $1, e, e^{2}, \ldots, e^{3 m-1}$, in which we have the relation $e^{3 m}=\sum_{i=0}^{3 m-1} w_{3 m-i}(\beta) \cdot e^{i}$. Since we have $w_{i}(\beta)=i^{*} w_{i}(\alpha)=0$ for $0<i<2 m$ by the assumption, we can write this relation as $e^{3 m}=w_{3 m}+w_{3 m-1} \cdot e+\cdots+w_{2 m}$ - $e^{m}$, where we have abbreviated $w_{i}(\beta)$ as $w_{i}$. We apply the total squaring operation $\mathrm{Sq}=\sum_{i \geqslant 0} \mathrm{Sq}^{i}$ to this relation. Since $\mathrm{Sq}\left(e^{i}\right)=(\mathrm{Sq} e)^{i}=\left(e+e^{2}\right)^{i}=e^{i}(1+e)^{i}$, we obtain the following equation:

$$
e^{3 m}(1+e)^{3 m}=\mathrm{Sq} w_{3 m}+\mathrm{Sq} w_{3 m-1} \cdot e(1+e)+\cdots+\mathrm{Sq} w_{2 m} \cdot e^{m}(1+e)^{m} .
$$

In this equation, we like to compare the coefficients of $e^{j}$ 's. To do this, we must rewrite the left-hand side of (***) so that all summands have exponents of $e$ less than $3 \mathrm{~m}$. We calculate using the previous relation as follows:

$$
\begin{aligned}
e^{3 m}(1+e)^{3 m}= & e^{3 m}\left(1+e^{m}+e^{2 m}+e^{3 m}\right) \\
= & e^{3 m}\left(1+e^{m}\right)+\left(e^{3 m}-w_{2 m} \cdot e^{m}\right) e^{2 m}+w_{2 m} \cdot e^{3 m}+\left(e^{3 m}\right)^{2} \\
= & \left(w_{3 m}+w_{3 m-1} \cdot e+\cdots+w_{2 m} \cdot e^{m}\right)\left(1+e^{m}\right) \\
& +\left(w_{3 m}+w_{3 m-1} \cdot e+\cdots+w_{2 m+1} \cdot e^{m-1}\right) e^{2 m} \\
& +w_{2 m}\left(w_{3 m}+w_{3 m-1} \cdot e+\cdots+w_{2 m} \cdot e^{m}\right) \\
& +\left(w_{3 m}+w_{3 m-1} \cdot e+\cdots+w_{2 m} \cdot e^{m}\right)^{2} .
\end{aligned}
$$


With this expression of the left-hand side of $(* * *)$, we can compare the coefficients of $e^{j}$ 's for $j<3 m$. Comparing the coefficients of $e^{2 m}$, we obtain $\mathrm{Sq} w_{2 m}=w_{2 m}$ $+w_{3 m}+w_{2 m}^{2}$. Hence we have $\mathrm{Sq}^{j} w_{2 m}=0$ for $0<j<m$ and $\mathrm{Sq}^{m} w_{2 m}=w_{3 m}$. We here recall that $w_{i}=i^{*} w_{i}(\alpha)$. Since $i^{*}: H^{i}(B) \rightarrow H^{i}\left(B^{(3 m)}\right)$ is monomorphic for $i \leqslant 3 m$, we conclude that $\operatorname{Sq}^{j} w_{2 m}(\alpha)=0$ for $0<j<m$ and $\operatorname{Sq}^{m} w_{2 m}(\alpha)=w_{3 m}(\alpha)$. Thus the lemma follows.

Remark. When $w_{i}=0$ for $0<i<2^{r}$, Wu's formula [10] turns out to be $\mathrm{Sq}^{j} w_{2^{r}}$ $=\left(\begin{array}{c}2^{r}-1 \\ j\end{array}\right) w_{2^{r}+j}=w_{2^{r}+j}\left(0<j<2^{r}\right)$. Lemma 3.3 implies that this is zero for $0<j$ $<2^{r-1}$. We also remark that there is a vector bundle over $\Sigma^{4} \mathbb{H} P^{2}$ such that $w_{8} \neq 0$ and $w_{12} \neq 0$ (see [8, Theorem 4.5]). Thus our result is best possible at least for $r=3$.

Now, we consider the second case: $k=3,5$ or 7 . The result is as follows.

Proposition 3.4. Let $k=3,5$ or 7. Then $\Sigma^{k} \mathbb{R} P^{n}$ is not $W$-trivial if and only if $n+k=4$ or 8 .

Proof. We consider the cofibration $\Sigma^{k} \mathbb{R} P^{n-1} \stackrel{i}{\rightarrow} \Sigma^{k} \mathbb{R} P^{n} \stackrel{j}{\rightarrow} S^{n+k}$. First, let $n+k$ $=8$. Since $S^{8}$ is not W-trivial and $j^{*}: H^{8}\left(S^{8}\right) \rightarrow H^{8}\left(\Sigma^{k} \mathbb{R} P^{n}\right)$ is monomorphic, it follows from Lemma 1.8 that $\Sigma^{k} \mathbb{R} P^{n}$ is not W-trivial. Similarly $\Sigma^{k} \mathbb{R} P^{n}$ is not W-trivial when $n+k=4$. Thus the "if" part of the proposition follows. Next, we suppose $n+k \neq 4,8$ and show that $\Sigma^{k} \mathbb{R} P^{n}$ is $\mathrm{W}$-trivial. Our proof is divided into two cases.

Case 1: $n+k \geqslant 16$.

First we consider the case when $n+k=2^{r}$ with $r \geqslant 4$. In this case, we have $\widetilde{K O}\left(\Sigma^{k} \mathbb{R} P^{n-1}\right)=0$ by [3, Theorem 1] since $k=3,5,7$ and $n+k-1 \equiv 7(\bmod 8)$. Hence $j^{*}: \widetilde{K O}\left(S^{2^{r}}\right) \rightarrow \widetilde{K O}\left(\Sigma^{k} \mathbb{R} P^{n}\right)$ is epimorphic. Since $S^{2^{r}}$ is W-trivial for $r \geqslant 4$, it follows from Lemma 1.7 that $\Sigma^{k} \mathbb{R} P^{n}$ is W-trivial, that is, $\Sigma^{k} \mathbb{R} P^{2^{r}-k}$ is $\mathrm{W}$-trivial for all $r \geqslant 4$. Hence, by Lemma 3.2, we see that $\Sigma^{k} \mathbb{R} P^{n}$ is W-trivial for all $n \geqslant 16-k$.

Case 2: $k+1 \leqslant n+k<16(n+k \neq 4,8)$.

Let $\alpha$ be a vector bundle over $\Sigma^{k} \mathbb{R} P^{n}$ and let $r$ be the smallest integer such that $w_{2^{r}}(\alpha)$ is (possibly) non-zero. Then we obviously have $r=2$ or 3 when $k=3$, and $r=3$ when $k=5,7$. Also, note that $2^{r}<n+k$ from our assumption $n+k \neq 4,8$. From Lemma 3.3, we must have $\operatorname{Sq}^{1} w_{2^{r}}(\alpha)=0$. On the other hand, since $k$ is odd and $2^{r}<n+k, \mathrm{Sq}^{1}: H^{2^{r}}\left(\Sigma^{k} \mathbb{R} P^{n}\right) \rightarrow H^{2^{r}+1}\left(\Sigma^{k} \mathbb{R} P^{n}\right)$ is non-trivial. Therefore, we have $w_{2^{r}}(\alpha)=0$. We thus obtain $W(\alpha)=1$ and conclude that $\Sigma^{k} \mathbb{R} P^{n}$ is $\mathrm{W}$-trivial if $n+k<16(n+k \neq 4,8)$. This completes the proof of the proposition.

Finally, we consider the third case: $k=6$. The result is as follows.

Proposition 3.5. $\Sigma^{6} \mathbb{R} P^{n}$ is not $W$-trivial if and only if $n=2$ or 3 .

Proof. The proof is very similar to that of the preceding proposition. Considering the cofibration $S^{7} \stackrel{i}{\longrightarrow} \Sigma^{6} \mathbb{R} P^{2} \stackrel{j}{\longrightarrow} S^{8}$, we see that $\Sigma^{6} \mathbb{R} P^{2}$ is not W-trivial in exactly the same way as before. Let us consider the cofibration $\Sigma^{6} \mathbb{R} P^{2} \stackrel{i}{\longrightarrow} \Sigma^{6} \mathbb{R} P^{3} \stackrel{j}{\longrightarrow} S^{9}$. Since $\widetilde{K O}\left(\Sigma^{6} \mathbb{R} P^{2}\right)$ is a finite group (precisely, $\mathbb{Z}_{2}$ ), we see from the exact sequence that $i^{*}: \widetilde{K O}\left(\Sigma^{6} \mathbb{R} P^{3}\right) \rightarrow \widetilde{K O}\left(\Sigma^{6} \mathbb{R} P^{2}\right)$ is epimorphic. Since $\Sigma^{6} \mathbb{R} P^{2}$ is not W-trivial, as shown above, it follows from Lemma 1.7 that $\Sigma^{6} \mathbb{R} P^{3}$ is not $\mathrm{W}$-trivial either. Thus 
the "if" part of the proposition follows. Next, we show that $\Sigma^{6} \mathbb{R} P^{n}$ is W-trivial for $n \neq 2,3$.

Case 1: $n \geqslant 10$.

By [3, Theorem 1], we have $\widetilde{K O}\left(\Sigma^{6} \mathbb{R} P^{n-1}\right)=0$ when $n+6=2^{r}(r \geqslant 4)$. Hence $j^{*}: \widetilde{K O}\left(S^{2^{r}}\right) \rightarrow \widetilde{K O}\left(\Sigma^{6} \mathbb{R} P^{n}\right)$ is epimorphic, from which we see by Lemma 1.7 that $\Sigma^{6} \mathbb{R} P^{n}$ is W-trivial when $n+6=2^{r}(r \geqslant 4)$. Therefore, it follows from Lemma 3.2 that $\Sigma^{6} \mathbb{R} P^{n}$ is W-trivial for all $n \geqslant 10$.

Case 2: $1 \leqslant n<10(n \neq 2,3)$.

Obviously $\Sigma^{6} \mathbb{R} P^{n}$ is W-trivial when $n=1$. So we suppose that $n \geqslant 4$. For a vector bundle $\alpha$ over $\Sigma^{6} \mathbb{R} P^{n}$, the smallest integer such that $w_{2^{r}}(\alpha)$ is (possibly) nonzero is 8. Hence, from Lemma 3.3, we have $\mathrm{Sq}^{j} w_{8}(\alpha)=0$ for $0<j<4$. Since $\mathrm{Sq}^{1}$ acts trivially on $H^{8}\left(\Sigma^{6} \mathbb{R} P^{n}\right)$, we use $\mathrm{Sq}^{2}$ in place of $\mathrm{Sq}^{1}$. Indeed, $\mathrm{Sq}^{2}: H^{8}\left(\Sigma^{6} \mathbb{R} P^{n}\right)$ $\rightarrow H^{10}\left(\Sigma^{6} \mathbb{R} P^{n}\right)$ is non-trivial since $n \geqslant 4$. Therefore, we have $w_{8}(\alpha)=0$, so that we obtain $W(\alpha)=1$. Thus $\Sigma^{6} \mathbb{R} P^{n}$ is $\mathrm{W}$-trivial when $4 \leqslant n<10$. This completes the proof of the proposition.

The proof of Theorem 1.4 is completed by Propositions 3.1, 3.4 and 3.5.

\section{Proof of Theorem $\mathbf{1 . 6}$}

In this section, we investigate whether or not $\Sigma^{k} F P^{n}$ is $\mathrm{W}$-trivial for $F=\mathbb{H}$. Because of Corollary 1.2, we have only to consider the case when $0<k \leqslant 8$. Then, unless $k=4$ or $8, \Sigma^{k} \mathbb{H} P^{n}$ has no cells of dimension a power of 2 , so that we have $H^{2^{r}}\left(\Sigma^{k} \mathbb{H} P^{n}\right)=0$ for all $r \geqslant 0$. Thus, from Lemma 1.8 , the possibility for $\Sigma^{k} \mathbb{H} P^{n}$ not to be $\mathrm{W}$-trivial is only when $k=4$ or 8 . Therefore, Theorem 1.6 follows if we prove the following proposition.

\section{Proposition 4.1.}

(1) $\Sigma^{4} \mathbb{H} P^{n}$ is not $W$-trivial for all $n>1$.

(2) $\Sigma^{8} \mathbb{H} P^{n}$ is $W$-trivial for all $n>1$.

Proof. First, let us consider the cofibration $S^{8} \stackrel{i}{\longrightarrow} \Sigma^{4} \mathbb{H} P^{n} \stackrel{j}{\longrightarrow} \Sigma^{4}\left(\mathbb{H} P^{n} / S^{4}\right)$. Since $\Sigma^{3}\left(\mathbb{H} P^{n} / S^{4}\right)$ has cells only of dimension 3 or 7 modulo 8 , we have $\widetilde{K O}\left(\Sigma^{3}\left(\mathbb{H} P^{n} / S^{4}\right)\right)$ $=0$ from the Atiyah-Hirzebruch spectral sequence $[\mathbf{2}]$. Hence, $i^{*}: \widetilde{K O}\left(\Sigma^{4} \mathbb{H} P^{n}\right)$ $\rightarrow \widetilde{K O}\left(S^{8}\right)$ is epimorphic. Since $S^{8}$ is not W-trivial, it follows from Lemma 1.7 that $\Sigma^{4} \mathbb{H} P^{n}$ is not $\mathrm{W}$-trivial. This proves (1).

Next we prove (2). Let us consider the cofibration

$$
\Sigma^{8} \mathbb{H} P^{n-1} \stackrel{i}{\longrightarrow} \Sigma^{8} \mathbb{H} P^{n} \stackrel{j}{\longrightarrow} S^{4 n+8} .
$$

Since $\widetilde{K O}\left(S^{4 n+7}\right)=0, i^{*}: \widetilde{K O}\left(\Sigma^{8} \mathbb{H} P^{n}\right) \rightarrow \widetilde{K O}\left(\Sigma^{8} \mathbb{H} P^{n-1}\right)$ is epimorphic. Hence, we see that if $\Sigma^{8} \mathbb{H} P^{n}$ is W-trivial, then $\Sigma^{8} \mathbb{H} P^{n-1}$ is also W-trivial. Thus, it suffices to prove that $\Sigma^{8} \mathbb{H} P^{2^{m}}$ is W-trivial for all $m \geqslant 3$. Now, let $\alpha$ be a vector bundle over $\Sigma^{8} \mathbb{H} P^{2^{m}}$. Abusing notation, let $i$ denote the inclusion $\Sigma^{8} \mathbb{H} P^{2} \hookrightarrow \Sigma^{8} \mathbb{H} P^{2^{m}}$. From [7, Theorem 4.3], $\Sigma^{8} \mathbb{H} P^{2}$ is W-trivial. Since $i^{*}: H^{16}\left(\Sigma^{8} \mathbb{H} P^{2^{m}}\right) \rightarrow H^{16}\left(\Sigma^{8} \mathbb{H} P^{2}\right)$ is monomorphic, we obtain $w_{16}(\alpha)=0$. Let $r$ be the smallest integer such that $w_{2^{r}}(\alpha)$ is (possibly) non-zero. Then, we have $r \geqslant 5$ from the above argument. Also note 
that $r \leqslant m+2$ since $2^{r} \leqslant 8+4 \cdot 2^{m}$ and $r \geqslant 5$. Now let us consider the operation $\mathrm{Sq}^{8}: H^{2^{r}}\left(\Sigma^{8} \mathbb{H} P^{2^{m}}\right) \rightarrow H^{2^{r}+8}\left(\Sigma^{8} \mathbb{H} P^{2^{m}}\right)$. Since $\left(\begin{array}{c}2^{r-2}-2 \\ 2\end{array}\right) \equiv 1(\bmod 2)$ and $r \leqslant m+2$, this operation is non-trivial. On the other hand, by Lemma 3.3, we have $\mathrm{Sq}^{8} w_{2^{r}}(\alpha)$ $=0$ since $r \geqslant 5$. Therefore, we obtain $w_{2^{r}}(\alpha)=0$ and conclude that $W(\alpha)=1$. This completes the proof.

\section{Proof of Theorem 1.5}

Finally, in this section, we investigate whether $\Sigma^{k} \mathbb{C} P^{n}$ is W-trivial or not. If $k$ is odd, then $\Sigma^{k} \mathbb{C} P^{n}$ has no cells of dimension a power of 2 . Thus, from Lemma 1.8 and Corollary 1.2 , the possibility of $\Sigma^{k} \mathbb{C} P^{n}$ being not $\mathrm{W}$-trivial is only when $k=2,4,6$ or 8 . For $k=2$ or 4 , we have the following result.

Proposition 5.1. $\Sigma^{2} \mathbb{C} P^{n}$ and $\Sigma^{4} \mathbb{C} P^{n}$ are not $W$-trivial for all $n>1$.

Proof. First we consider $\Sigma^{2} \mathbb{C} P^{n}$. Analogously to the proof of Proposition 3.1, we consider the exact sequence

$$
0 \longleftarrow \widetilde{K}\left(S^{2} \vee \mathbb{C} P^{n}\right) \stackrel{i^{*}}{\longleftarrow} \widetilde{K}\left(S^{2} \times \mathbb{C} P^{n}\right) \stackrel{j^{*}}{\longleftarrow} \widetilde{K}\left(\Sigma^{2} \mathbb{C} P^{n}\right) \longleftarrow 0
$$

and the stable class of $\left(p_{1}^{*} \nu-1\right) \otimes_{\mathbb{C}}\left(p_{2}^{*} \eta-1\right)$. Here, $\nu$ is the Hopf vector bundle over $S^{2}$ considered as a complex (line) bundle, while $\eta$ is the canonical complex line bundle over $\mathbb{C} P^{n}$. Then, we can take a complex vector bundle $\alpha$ over $\Sigma^{2} \mathbb{C} P^{n}$ such that $j^{*} \alpha$ is stably equivalent to $\left(p_{1}^{*} \nu-1\right) \otimes_{\mathbb{C}}\left(p_{2}^{*} \eta-1\right)$. By Lemma 2.1 , in $H^{*}\left(S^{2} \times \mathbb{C} P^{n} ; \mathbb{Z}\right)$,

$$
\begin{aligned}
C\left(j^{*} \alpha\right) & =C\left(\left(p_{1}^{*} \nu-1\right) \otimes_{\mathbb{C}}\left(p_{2}^{*} \eta-1\right)\right) \\
& =1+c_{1}(\nu) \times\left(\left(1+c_{1}(\eta)\right)^{-1}-1\right) \\
& =1+s \times\left(-t+t^{2}-t^{3}+\cdots\right),
\end{aligned}
$$

where $s$ and $t$ are generators of $H^{2}\left(S^{2} ; \mathbb{Z}\right)$ and $H^{2}\left(\mathbb{C} P^{n} ; \mathbb{Z}\right)$, respectively. Hence we have $j^{*} c_{2}(\alpha)=-s \times t \not \equiv 0(\bmod 2)$ for $n \geqslant 1$. Therefore we have $w_{4}(\alpha) \neq 0$, so that $\Sigma^{2} \mathbb{C} P^{n}$ is not $\mathrm{W}$-trivial for $n \geqslant 1$.

Similarly for $\Sigma^{4} \mathbb{C} P^{n}$, let us consider the exact sequence

$$
0 \longleftarrow \widetilde{K}\left(S^{4} \vee \mathbb{C} P^{n}\right) \stackrel{i^{*}}{\longleftarrow} \widetilde{K}\left(S^{4} \times \mathbb{C} P^{n}\right) \stackrel{j^{*}}{\longleftarrow} \widetilde{K}\left(\Sigma^{4} \mathbb{C} P^{n}\right) \longleftarrow 0
$$

Let $\nu_{2}$ be a complex vector bundle whose stable class is a generator of $\widetilde{K}\left(S^{4}\right)$. We can take $\nu_{2}$ as $\operatorname{dim}_{\mathbb{C}} \nu_{2}=2$. From the previous argument, considering $S^{4}$ as $\Sigma^{2} \mathbb{C} P^{1}$, we see that $c_{2}\left(\nu_{2}\right)=-s_{2}$, where $s_{2}$ is the generator of $H^{4}\left(S^{4} ; \mathbb{Z}\right)$ corresponding to $s \times t$. Now we take a complex vector bundle $\alpha$ over $\Sigma^{4} \mathbb{C} P^{n}$ such that $j^{*} \alpha$ is stably equivalent to $\left(p_{1}^{*} \nu_{2}-2\right) \otimes_{\mathbb{C}}\left(p_{2}^{*} \eta-1\right)$. By Lemma 2.1 , in $H^{*}\left(S^{4} \times \mathbb{C} P^{n} ; \mathbb{Z}\right)$ we have

$$
\begin{aligned}
C\left(j^{*} \alpha\right) & =C\left(\left(p_{1}^{*} \nu_{2}-2\right) \otimes_{\mathbb{C}}\left(p_{2}^{*} \eta-1\right)\right) \\
& =1+c_{2}\left(\nu_{2}\right) \times\left(\left(1+c_{1}(\eta)\right)^{-2}-1\right) \\
& =1-s_{2} \times\left(-2 t+3 t^{2}-4 t^{3}+\cdots\right) .
\end{aligned}
$$

Hence we have $j^{*} c_{4}(\alpha)=-3 s_{2} \times t^{2} \not \equiv 0(\bmod 2)$ for $n \geqslant 2$. Therefore we have $w_{8}(\alpha)$ $\neq 0$, so that $\Sigma^{4} \mathbb{C} P^{n}$ is not $\mathrm{W}$-trivial for $n \geqslant 2$. 
Here, before we proceed to consider $\Sigma^{k} \mathbb{C} P^{n}$ for $k=6$ or 8 , we need to prepare a lemma concerning Steenrod operations. For a non-negative integer $m$, let $\alpha(m)$ denote the number of ones in the dyadic expansion of $m$. It is easy to see that if $m$ and $\ell$ are positive integers such that $\left(\begin{array}{c}m \\ \ell\end{array}\right) \equiv 1(\bmod 2)$, then $\alpha(m+\ell) \leqslant \alpha(m)$. Also, we clearly have $\alpha\left(2^{\ell+1}-k\right)=\alpha\left(2^{\ell}-k\right)+1$ for any integer $k$ with $0<k \leqslant 2^{\ell}$, whence we have $\alpha\left(2^{r-1}-k\right)>\alpha\left(2^{j-1}-k\right)$ for positive integers $j$ and $r$ with $j<r$. Thus, we obtain the following lemma.

Lemma 5.2. If $k>0$, any Steenrod operation $\varphi: H^{2^{j}}\left(\Sigma^{2 k} \mathbb{C} P^{n}\right) \rightarrow H^{2^{r}}\left(\Sigma^{2 k} \mathbb{C} P^{n}\right)$ is trivial for $j<r$.

Now, we are ready to consider $\Sigma^{k} \mathbb{C} P^{n}$ for $k=6$ or 8 . We have the following result.

Proposition 5.3. $\Sigma^{6} \mathbb{C} P^{n}$ and $\Sigma^{8} \mathbb{C} P^{n}$ are $W$-trivial for all $n>1$.

Proof. Let $\alpha$ be a vector bundle over $\Sigma^{k} \mathbb{C} P^{n}$, where $k=6$ or 8 , and let $r$ be the smallest integer such that $w_{2^{r}}(\alpha)$ is (possibly) non-zero. Clearly we have $r \geqslant 3$ when $k=6$, and $r \geqslant 4$ when $k=8$. Since $\mathrm{Sq}^{2} w_{8}(\alpha)=0$ by Lemma 3.3 and also $\mathrm{Sq}^{2}: H^{8}\left(\Sigma^{6} \mathbb{C} P^{n}\right)$ $\rightarrow H^{10}\left(\Sigma^{6} \mathbb{C} P^{n}\right)$ is non-trivial, we have $w_{8}(\alpha)=0$. So we may suppose that $r \geqslant 4$ also when $k=6$. To prove $w_{2^{r}}(\alpha)=0$ for $r \geqslant 4$, the above method fails depending on the value of $n+k$. So we use secondary operations. Let $T(\alpha)$ be the Thom space of $\alpha$ and denote the Thom class by $U ; U \in H^{m}(D(\alpha), S(\alpha))=\widetilde{H}^{m}(T(\alpha))$, where $m=\operatorname{dim} \alpha$. Since $\mathrm{Sq}^{\ell} U=w_{\ell}(\alpha) U \quad(\ell>0)$, we have $\mathrm{Sq}^{\ell} U=0$ for $\ell<2^{r}$. Since $r \geqslant 4$, secondary operations on $U$ are defined. Indeed, for integers $i, j$ with $0 \leqslant i \leqslant j<r(i \neq j-1), \Phi_{i, j}(U) \in H^{m+d(i, j)}(T(\alpha))$ is defined with an indeterminacy $Q^{m+d(i, j)}(T(\alpha) ; i, j)$, where $d(i, j)=2^{i}+2^{j}-1$, and the following formula holds:

$$
\left[\mathrm{Sq}^{2^{r}} U\right]=\sum_{\substack{0 \leqslant i \leqslant j<r \\ i \neq j-1}} a_{i, j} \Phi_{i, j}(U) \text { modulo } \sum_{\substack{0 \leqslant i \leqslant j<r \\ i \neq j-1}} a_{i, j} Q^{m+d(i, j)}(T(\alpha) ; i, j),
$$

where each $a_{i, j}$ is a certain Steenrod operation (see [1, Theorem 4.6.1]). Now let us investigate each summand in this decomposition of $\mathrm{Sq}^{2} U$. We divide into two cases depending on whether $i$ is zero or not.

Case $1: i \neq 0$.

In this case, $d(i, j)$ is odd, so that we have $H^{d(i, j)}\left(\Sigma^{k} \mathbb{C} P^{n}\right)=0(k=6,8)$. Hence, by the Thom isomorphism, we have $H^{m+d(i, j)}(T(\alpha))=0$, so that $\Phi_{i, j}(U)=0$ and $Q^{m+d(i, j)}(T(\alpha) ; i, j)=0$.

Case 2: $i=0$.

In this case, $d(i, j)=2^{j}$. Therefore, it follows that $a_{i, j}$ is an operation $H^{m+2^{j}}(T(\alpha))$ $\rightarrow H^{m+2^{r}}(T(\alpha))$. We claim that the following diagram commutes, where the vertical maps are the Thom isomorphisms.

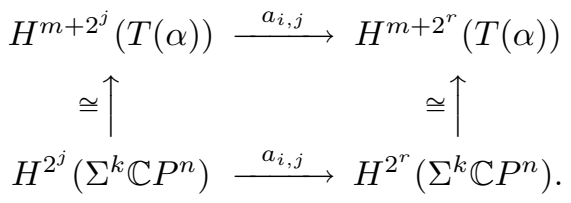

In fact, for $x \in H^{*}\left(\Sigma^{k} \mathbb{C} P^{n}\right)$ and $h \leqslant 2^{r}-2^{j}$, we have $\operatorname{Sq}^{h}(x U)=\operatorname{Sq}^{h} x \cdot U$ by the 
Cartan formula since $\mathrm{Sq}^{\ell} U=w_{\ell}(\alpha) U=0$ for $0<\ell<2^{r}$. Thus we have $a_{i, j}(x U)$ $=a_{i, j} x \cdot U$ for $x \in H^{2^{j}}\left(\Sigma^{k} \mathbb{C} P^{n}\right)$, so that the diagram commutes. Now, in the above diagram, the lower $a_{i, j}$ is trivial by Lemma 5.2. Therefore, we see that the upper $a_{i, j}$ is also trivial.

Therefore, from the arguments in Cases 1 and 2, we obtain $\left[\mathrm{Sq}^{2^{r}} U\right]=0$ modulo 0 , that is, $\mathrm{Sq}^{2^{r}} U=0$. Since $\mathrm{Sq}^{2^{r}} U=w_{2^{r}}(\alpha) U$, we conclude that $w_{2^{r}}(\alpha)=0$. This completes the proof of the proposition.

The proof of Theorem 1.5 is completed by Propositions 5.1 and 5.3.

\section{References}

[1] J.F. Adams, On the non-existence of elements of Hopf invariant one, Ann. of Math. 72 (1960), no. 1, 20-104.

[2] M.F. Atiyah and F. Hirzebruch, Vector bundles and homogeneous spaces, Proc. Sympos. Pure Math. III, Differential Geometry (1961), 7-38.

[3] M. Fujii, $K_{O}$-groups of projective spaces, Osaka J. Math. 4 (1967), no. 1, 141-149.

[4] F. Hirzebruch, Neue topologische Methoden in der algebraischen Geometrie, Springer-Verlag, New York, 1956.

[5] J. Milnor, Some consequences of a theorem of Bott, Ann. of Math. 68 (1958), no. $2,444-449$.

[6] R. Tanaka, On the index and co-index of sphere bundles, Kyushu J. Math. 57 (2003), no. 2, 371-382.

[7] R. Tanaka, A fiberwise analogue of the Borsuk-Ulam theorem for sphere bundles over a 2-cell complex II, Topology Appl. 154 (2007), no. 15, 2849-2855.

[8] R. Tanaka, On trivialities of Stiefel-Whitney classes of vector bundles over highly connected complexes, Topology Appl. 155 (2008), no. 15, 1687-1693.

[9] R. Tanaka, A Borsuk-Ulam type theorem for sphere bundles over stunted projective spaces, Topology Appl. 156 (2009), no. 5, 932-938.

[10] W.-t. Wu, Les $i$-carrés dans une variété grassmannienne, C.R. Acad. Sci. Paris 230 (1950), 918-920.

Ryuichi Tanaka tanaka_ryuichi@ma.noda.tus.ac.jp

Department of Mathematics, Faculty of Science and Technology, Tokyo University of Science, Noda, Chiba 278-8510, Japan 